\title{
REHOVOT RADIOCARBON MEASUREMENTS II
}

A. KAUFMAN, I. CARMII, and R. SCHLESINGER

The Weizmann Institute of Science, Rehovot, Israel

This list contains results obtained since July 1970. They are mainly hydrologic and geologic samples prepared and counted as described in our previous date list, (Carmi et al., 1971). The coordinate system used to describe sampling locations is the local one.

\section{SAMPLE DESCRIP'TIONS}

\section{HYDROIOGIC SAMPLFS}

Samples from the Galilee were subm. by E. Wackshall, Helorew Univ.; those from Samaria by E. Rosenthal, Geol. Inst., and G. Dror, Hydrol. Service; those from the Judean Desert by U. Baida, Water Planning Comm. for Isracl, Ltd.; those from Chamat Gader and Sample RT-307E by E. Mazor, Weizmann Inst., and Sample RT-322 by A. Issar, Geol. Inst. This last sample is much less active than the shallow Sample $\mathrm{RT}$-140 in our previous clate list. $\delta \mathrm{C}^{13}$ values, relative to $\mathrm{PDB}$, were measured to permit apparent age estimation according to Ingerson and Pearson (1964). These ages are not given due to the uncertain validity of their assumptions.

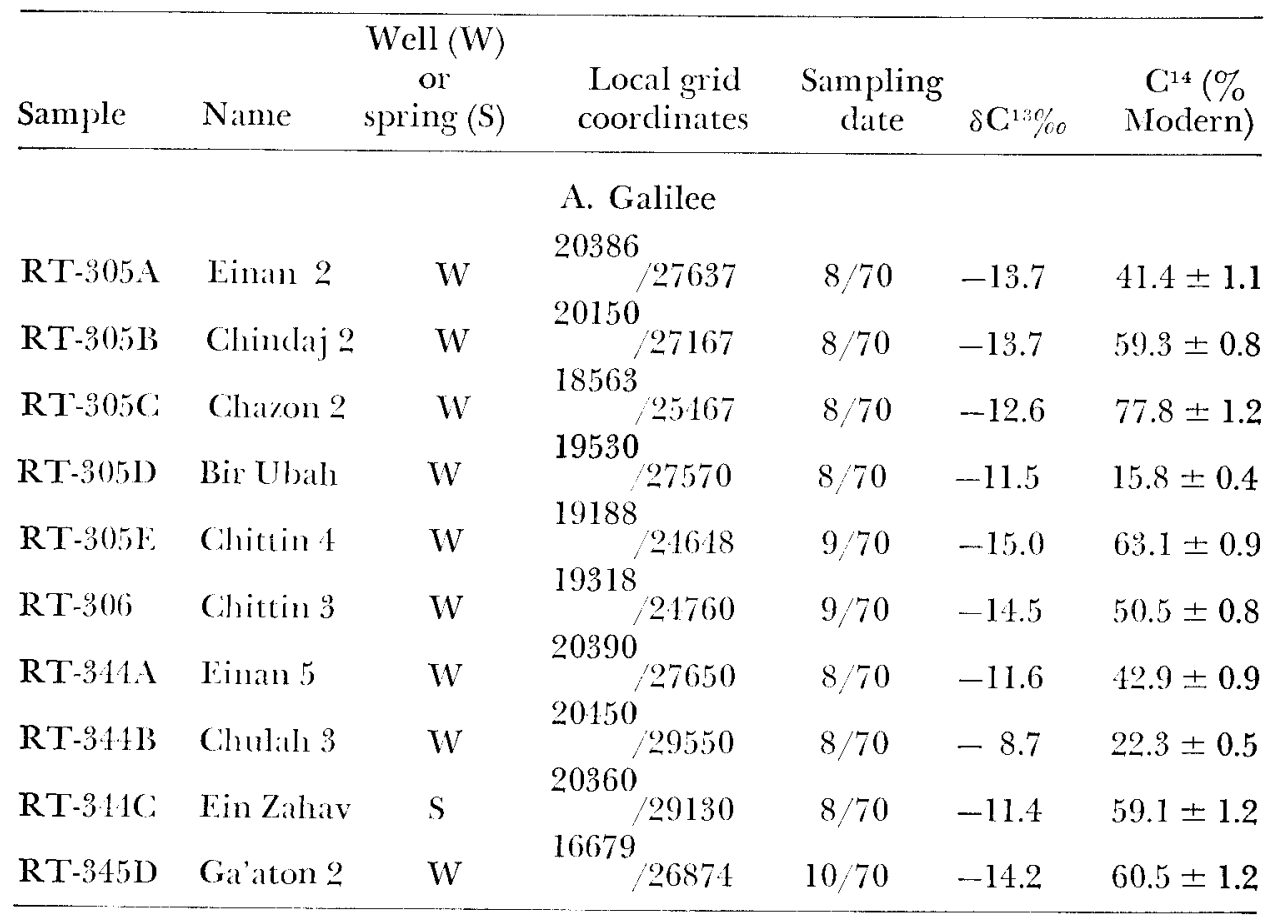




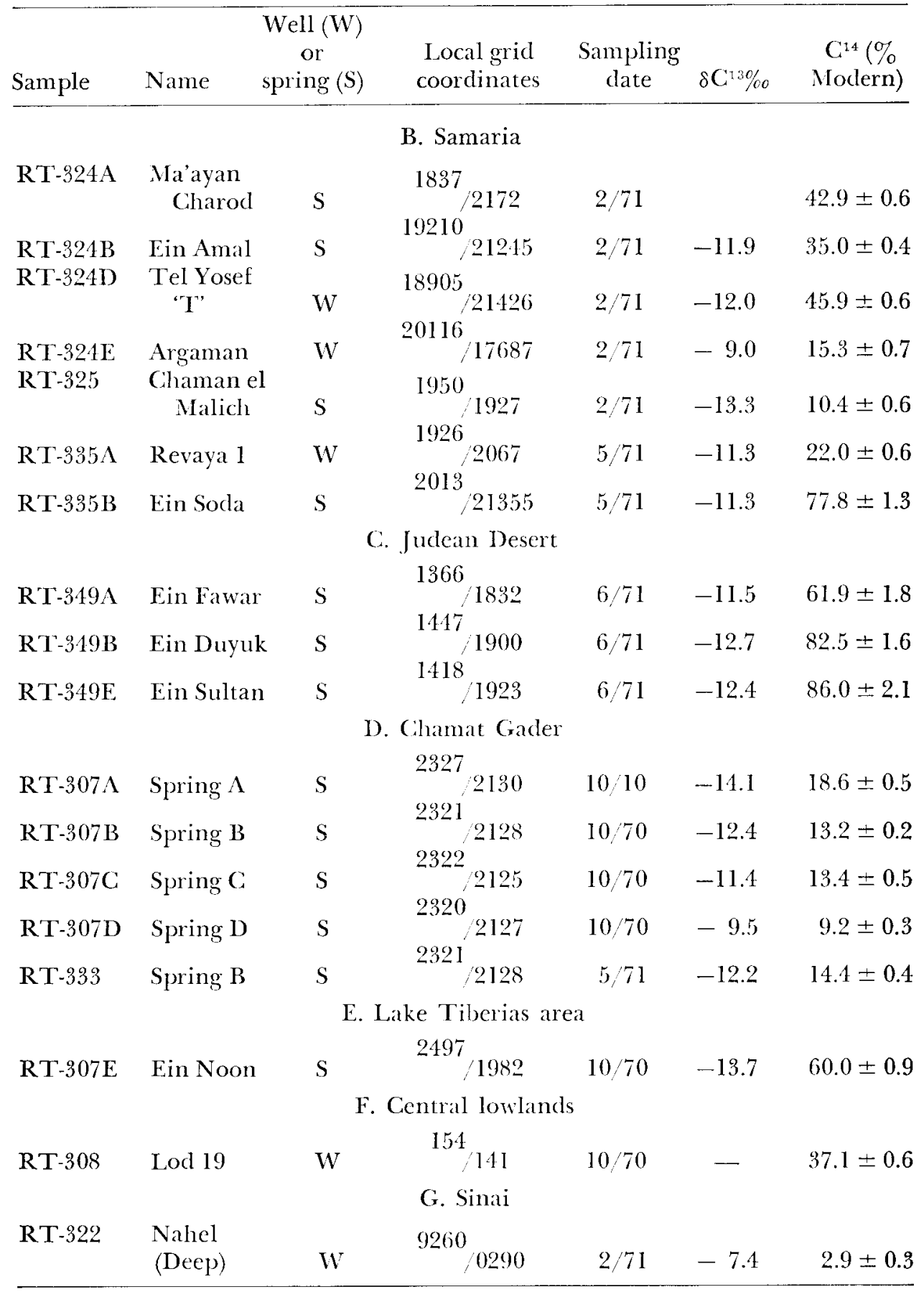


II. GEOLOGIC SAMPLIS

\section{A. Lake Kinneret cores}

Two cores were coll. from lake bottom sediments by M. Stiller. Core 'D' coll. Nov. 1969 in $42 \mathrm{~m}$ water at position $2467 / 2070$, and Core 'F' Oct. 1970 in $25 \mathrm{~m}$ water at position includes $50 \%$ carbonate and 20 organic matter; these 2 phases were run separately.

\begin{tabular}{|c|c|c|c|c|c|c|c|}
\hline \multirow[b]{2}{*}{ Core } & \multirow[b]{2}{*}{ Interval } & \multicolumn{3}{|c|}{ Organic fraction } & \multicolumn{3}{|c|}{ Inorganic fraction } \\
\hline & & Sample & $\delta(136 / 00$ & $\begin{array}{c}\mathrm{C}^{14}(\%) \\
\text { Modern) }\end{array}$ & Sample & $\delta \mathrm{C}^{13 \%} \%$ & $\begin{array}{r}\mathrm{C}^{14}(\% \\
\text { Modern })\end{array}$ \\
\hline $\mathrm{D}$ & $0-8 \mathrm{~cm}$ & RT-225 & - & $63.8 \pm 3.1$ & RT-224 & - & $74.2 \pm 1.1$ \\
\hline D & $16.5-$ & & & & & & \\
\hline & $24.5 \mathrm{~cm}$ & R I -219 & - & $84.3 \pm 3.6$ & RT-218 & - & $73.2 \pm 1.0$ \\
\hline $\mathrm{D}$ & $32.42 \mathrm{~cm}$ & $\mathrm{RT}-229$ & — & $75.3 \pm 5.2$ & R'I'-222 & - & $35.3 \pm 0.7$ \\
\hline $\mathrm{F}$ & $0-10 \mathrm{~cm}$ & RT'-318D-ORG & -29.1 & $76.3 \pm 2.0$ & RT $-318 \mathrm{I}$ & -2.4 & $80.7 \pm 1.2$ \\
\hline $\mathrm{F}$ & $10.20 \mathrm{~cm}$ & RT'-318A-ORG; & -30.7 & $63.9 \pm 1.4$ & $\mathrm{RT}^{\prime}-318 \mathrm{~A}$ & -2.2 & $69.2 \pm 1.0$ \\
\hline $\mathrm{F}$ & $20-30 \mathrm{~cm}$ & RI-318B-ORG & -30.8 & $94.5 \pm 3.7$ & RT-318B & -2.7 & $74.3 \pm 1.1$ \\
\hline F & $30-40 \mathrm{~cm}$ & RT-318C-ORG & -30.2 & $89.1 \pm 2.8$ & $\mathrm{R}^{\prime} \mathrm{T}-318 \mathrm{C}$ & -1.0 & $78.2 \pm 1.7$ \\
\hline $\mathrm{F}$ & $40-50 \mathrm{~cm}$ & RT-315-ORG & -30.7 & $85.9 \pm 2.4$ & $\mathrm{RT}^{\prime}-315$ & -1.4 & $75.8 \pm 1.2$ \\
\hline
\end{tabular}

B. Secondary calciles

Results on other samples from lst 3 series mentioned here (Qsalon, Sha'ar Fagai, and IIermon) appear in Carmi el al. (1971). Samples coll. by M. Magaritz, March 1970 to Oct. 1970, except for N'tiv Halamed Hai series which was coll. June 1971. Ages are calculated assuming precipitations from bicarbonate solutions whose carbon is lerived from soil $\mathrm{CO}\left(\%(\mathrm{C})=100, \delta(13-25 \%)\right.$ and country rock $\% \mathrm{C}^{14}=0, \delta \mathrm{C}^{13}=$ as measured). In each case, $\delta C^{13}$ of the country rock is given (see p. 182-3).

RT-321A.

\section{Dead Sea area carbonales}

$10,700 \pm 150$

Travertine from hillside above Ein Fescha $\left(\begin{array}{l}1247 \\ 1925\end{array}\right)$, coll. 1969 by F. Yaron.

\section{RT-321B.}

$$
\begin{array}{r}
4200 \pm 150 \\
\delta C^{1 s}=+1.3 \%
\end{array}
$$

Travertine coll. 2 m from RT-321A.

RT-342B.

$$
\begin{array}{r}
\mathbf{3 8 0 0} \pm \mathbf{2 4 0} \\
\delta C^{13}=+0.4 \%
\end{array}
$$

Precipitated carbonate crust from cliff overlooking Ras Fescha $(1231 / 1927)$ at $-392 \mathrm{~m}$, coll. July $197 \mathrm{l}$ by $\mathrm{C}$. Klein. 


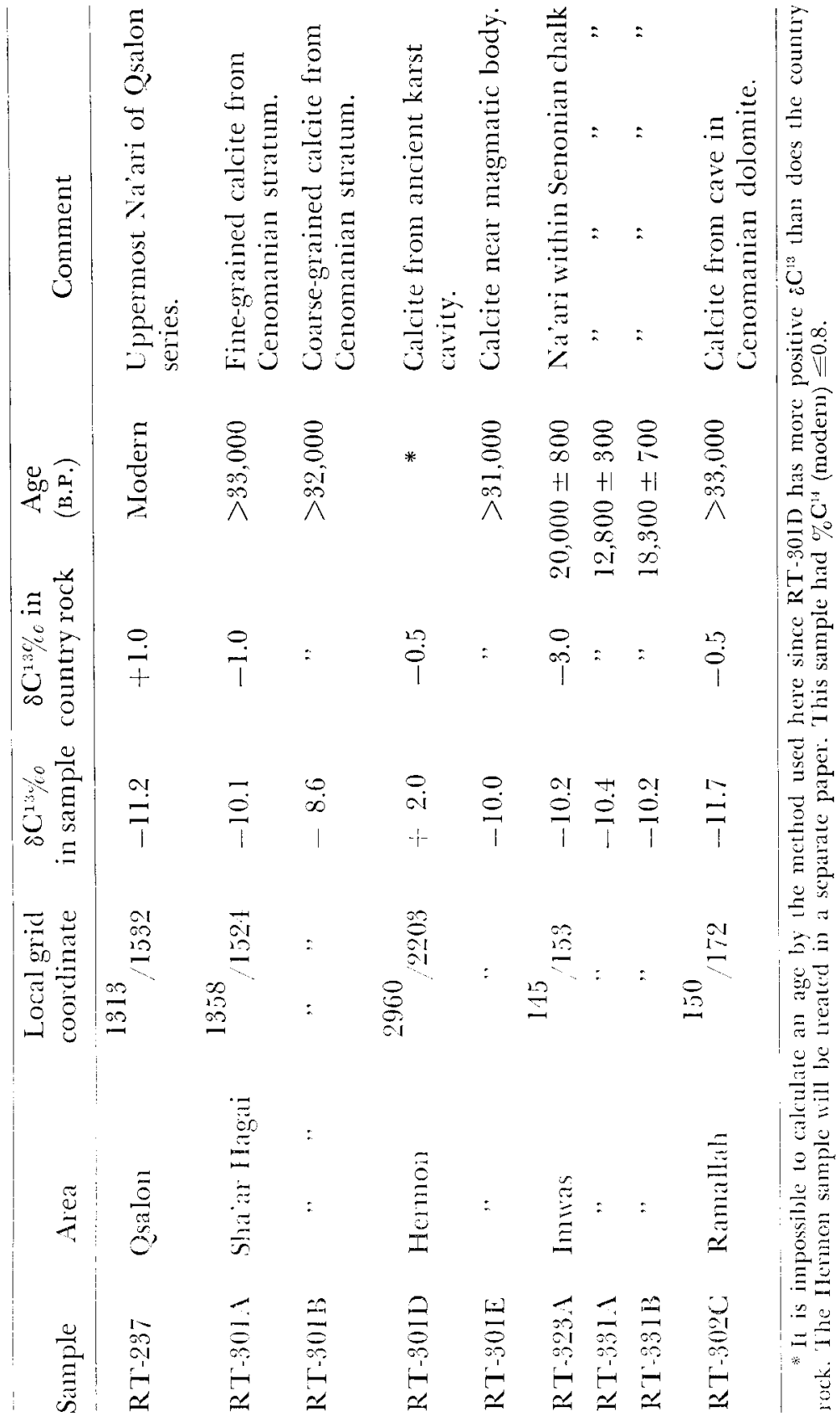




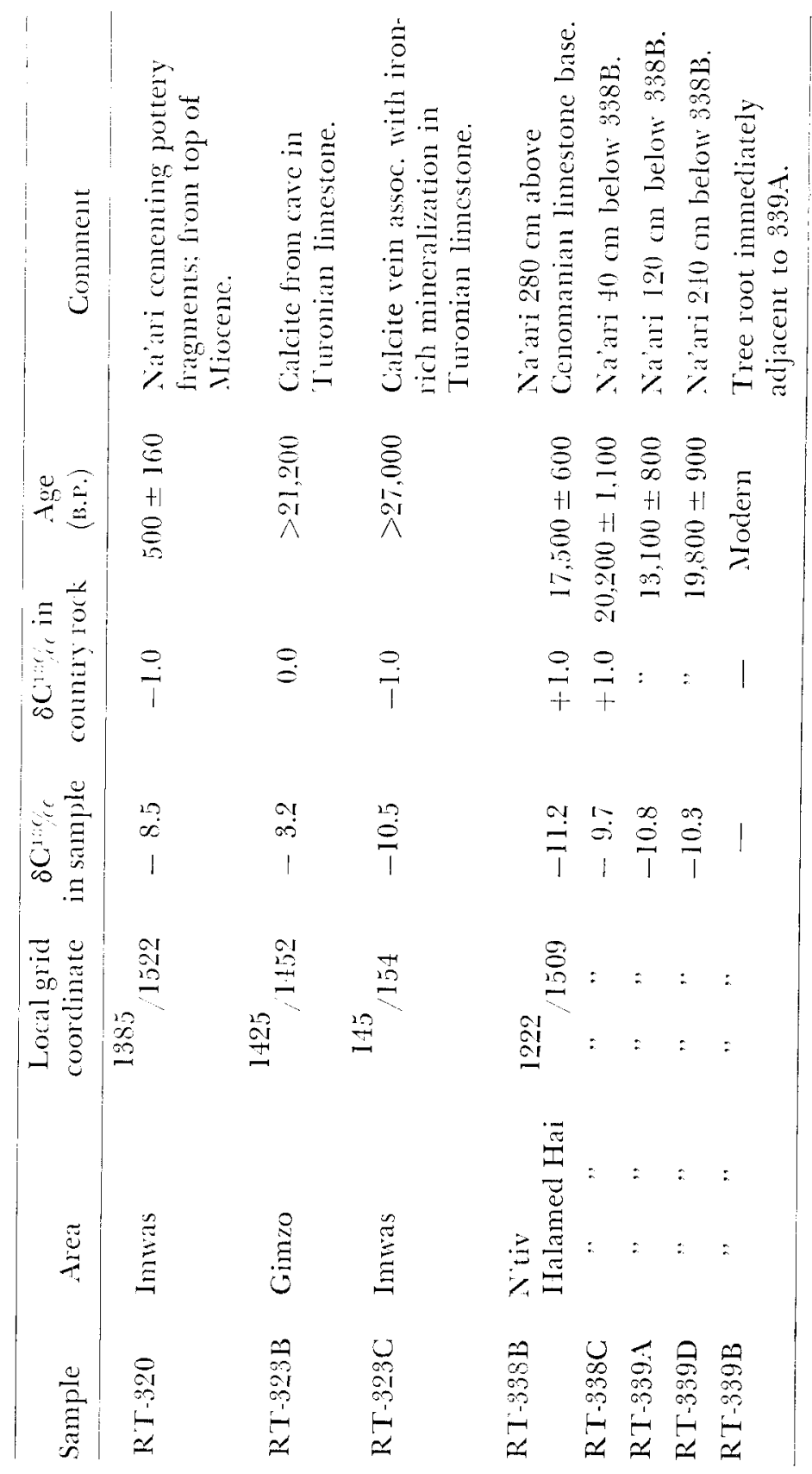


RT-319.

Oolites from Ras Matarma lagoon, N Gulf of Suez, $\left(\begin{array}{l}876 \\ / 930\end{array}\right)$, subm. Dec. 1970 by $A$. Nissenbaum.

\section{RT-348.}

Reef coral from erosion surface near Sharm-El-Sheik $\left(\begin{array}{c}698 \\ / 081\end{array}\right)$ coll. July 1971 by R. Garson.

III. ATMOSPHERIC SAMPLES

Two atmospheric CO, samples were absorbed with a concentrated $\mathrm{NaOH}$ solution. Comment (A.K.): results show confirmation of 1969 decrease noted in Rchovot atmosphere (Carmi et al., 1971) and a levelling off during 1970.

\section{RT-352A.}

$$
\delta \mathrm{C}^{14 \%}=\mathbf{5 0 . 3} \pm \mathbf{3 . 0}
$$

Exposed 26 Jan.-30 Jan. 1970.

\section{RT-352B.}

$$
\delta \mathrm{C}^{14 \%}=\mathbf{5 1 . 5} \pm \mathbf{3 . 0}
$$

Exposed 17 Jan.-24 Jan. 1971.

\section{REITRFNCES}

Carmi, I. Noter, Y., and Schlesinger, R., 197I, Rehovot radiocarbon measurements I: Radiocarbon, $v .13$, p. $412-419$.

Ingerson, E. and Pearson, F. J., J964, Estimation of ages and rate of motion of ground water, Recent researches in the ficld of hydrosphere, atmosphere and nuclear geochemistry: Tokyo, Maruzen Co., Ltel., p. 263-283. 